\title{
Review
}

\section{Present status of sentinel lymph node biopsy in cervical cancer}

\author{
Ariel Gustavo Glickman ${ }^{a, *}$, Sergio Valdes ${ }^{a}$, Blanca Gil-Ibañez ${ }^{a}$, \\ Pilar Paredes ${ }^{b}$, Karen Sttephannía Cortés ${ }^{c}$, Aureli Angel Torné Blade ${ }^{a}$
}

a Gynecological Oncology Unit, Barcelona Clinic Hospital, 170 Villaroel St, Barcelona 08036, Spain

b Nuclear Medicine, Barcelona Clinic Hospital, 170 Villaroel St, Barcelona 08036, Spain

${ }^{\mathrm{c}}$ Radiation Oncology, Barcelona Clinic Hospital, 170 Villaroel St, Barcelona 08036, Spain

\section{A R T I C L E I N F O}

\section{Article history:}

Received 18 November 2017

Received in revised form

17 February 2018

Accepted 8 April 2018

Available online 9 May 2018

\section{Keywords:}

Sentinel lymph node biopsy

Cervical cancer

Injection techniques and tracers

Detection rate

Sensitivity

Clinical relevance

\begin{abstract}
A B S T R A C T
Cervical cancer is the fourth most common cancer in women, and seventh overall. This disease represents a medical, economic and social burden. In early FIGO stage patients (IA, IB1 and IIA1), nodal involvement is the most important prognostic factor. Imaging evaluation of nodal metastasis is of limited value. In order to determine lymph node involvement, allow loco-regional control of the disease, define the need for adjuvant radiotherapy and improve survival, standard surgery for early disease is radical hysterectomy with systematic pelvic lymphadenectomy. However, this surgical treatment has risks and complications: longer operative time, larger blood loss, neurovascular or ureteral injury, lower-limb lymphedema, symptomatic lymphocysts, hydronephrosis. A method that allows to define the presence of regional metastasis with less morbidity and equal or greater precision is particularly relevant. The use of the sentinel lymph node biopsy is intended to reach that purpose. The present study reviews recent literature on the role of sentinel lymph node biopsy in cervical cancer, analyzing its indications and contraindications, injection and detection techniques, tracers used, surgical and pathological approaches and its applicability in up-to-date clinical practice.
\end{abstract}

๑ 2018 Greater Poland Cancer Centre. Published by Elsevier Sp. z o.o. All rights reserved.

\section{Background}

Cervical cancer is the fourth most common cancer in women, and seventh overall, with an estimate of 528,000 new cases in 2012 worldwide. A large majority occurs in the less developed regions (around $85 \%$ of them), where it accounts for almost $12 \%$ of all female cancers. There were an estimated 266,000 deaths from cervical cancer worldwide in 2012, accounting for $7.5 \%$ of all female cancer deaths. In Europe, its incidence shows wide differences, with very low rates in countries like Finland $(5 / 100,000)$ and much higher rates in Czech Republic $(18 / 100,000)$ or Romania $(30 / 100,000)$. This disease represents a medical, economic and social burden considering that $65 \%$ of the cases are diagnosed in women younger than 45 years of age. $^{1}$

\footnotetext{
* Corresponding author.

E-mail addresses: ariglickman@gmail.com (A.G. Glickman), sergiovaldes@gmail.com (S. Valdes), blgil@clinic.cat (B. Gil-Ibañez), pparedes@clinic.cat (P. Paredes), kscortes@clinic.cat (K.S. Cortés), atorne@clinic.cat (A.A. Torné Blade)

https://doi.org/10.1016/j.rpor.2018.04.004

1507-1367/@ 2018 Greater Poland Cancer Centre. Published by Elsevier Sp. z o.o. All rights reserved.
} 
Cervical cancer staging is based on the size of the primary tumor and anatomical structures affected. ${ }^{2}$ However, treatment election is based not only on the stage, but also on tumor parameters (histological type and grade, tumor size, surgical margins, depth of invasion, lymphovascular space invasion) and the presence of lymph node involvement. In early stage patients, nodal involvement is the most important prognostic factor. ${ }^{3}$ In fact, 5-year survival decreases from $88-92 \%$ to $55-64 \%$ in cases of positive pelvic nodes, despite Federation Internationale de Gynecologie et d'Obstetrique (FIGO) stage. ${ }^{4}$ Their number, location and size determine the prognosis and treatment of the primary tumor and the affected territories with chemo-radiotherapy. Nonetheless, latest FIGO staging from 2009 does not include lymph node status.

Following FIGO staging, we can define early cervical cancer as a tumor limited to the cervix, smaller than $4 \mathrm{~cm}$ (stages IA, IB1 and IIA1), without parametrial involvement and no suspicious images of regional disease. ${ }^{2}$ In the presence of histological diagnosis, staging is done through clinical examination and might be complemented by cone biopsy and radiodiagnostics techniques. ${ }^{5}$ When this group of patients present negative nodes, high survival rates is the rule and when they relapse, $66 \%$ of the cases do it in the pelvis. In contrast, when positive nodes are found their survival is significantly lower, and their relapses are only $55 \%$ in the pelvis. $^{6}$

Imaging evaluation of nodal metastasis is of limited value. The sensitivity of computed tomography (CT) scan and magnetic resonance imaging (MRI) are low for metastatic lymph node disease in patients with cervical cancer: $43 \%$ and $60 \%$, respectively. ${ }^{7}$ In patients with early stage cervical cancer, the sensitivity of positron emission tomography (PET)/CT to nodal involvement is also low (32-58\%), although the specificity is high $93-97 \%{ }^{8}$ Therefore, preoperative evaluation of nodal status with imaging techniques is not sensitive enough to replace the histological examination of dissected nodes. ${ }^{9}$

In order to determine lymph node involvement, allow loco-regional control of the disease, define the need for adjuvant radiotherapy and improve survival, standard surgery for early disease is radical hysterectomy with systematic pelvic lymphadenectomy. The approach could be either open, laparoscopic or robot-assisted laparoscopic. ${ }^{5}$ However, this surgical treatment has risks and complications. Patients who undergo radical surgery and lymphadenectomy may be exposed to longer operative time, larger blood loss, neurovascular or ureteral injury and also suffer a significant amount of complications in the long term: lower-limb lymphedema (10-15\%), symptomatic postoperative lymphocysts (12-34\%), hydronephrosis (4\%) and dysuria (20\%). ${ }^{9-12}$ Patients with early cervical cancer can present up to $27 \%$ positive nodes. ${ }^{13}$ Therefore, most of them have historically undergone unnecessary lymphadenectomies. ${ }^{9}$ Consequently, a method that allows to define the presence of regional metastasis with less morbidity and equal or greater precision is particularly relevant. The use of the sentinel lymph node (SLN) biopsy is intended to reach that purpose. The present study is a review of recent literature on the role of SLN biopsy in cervical cancer.

\section{Definition and indications}

The SLN is defined as the first node that drains a solid tumor; its status is considered to be representative of the local and regional lymph nodes status. The objective of SLN technique is to perform a selective biopsy while preserving the remaining lymph nodes in a way to limit morbidities, mostly those related to the lymphatic system. ${ }^{14}$ This procedure was first described by Cabanas forty years ago in patients with penile carcinoma. ${ }^{15}$ Gradually, it has become the standard of care for the management of melanoma, breast cancer and vulvar cancer, among other malignant lesions. The first experiences in cervical cancer were published at the beginning of the 2000s by O'Boyle et al. and Dargent et al. ${ }^{16,17}$; its feasibility has been broadly studied thereafter.

A trained multidisciplinary team, with a completed learning curve, should be involved in the realization of this technique. ${ }^{18}$ Even though the need for a learning curve has been enunciated since its development, the minimum number of cases required to validate SLN biopsy has not been determined yet. The tolerable margin for false-negative cases also remains irresolute. ${ }^{19}$ Khoury-Collado et al. established that 30 cases per surgeon are needed before achieving a good detection rate. Detection rate was defined as the percentage of procedures in which at least one sentinel node is identifiable. ${ }^{20}$

Current indications for SLN biopsy in cervical cancer comprise patients with early cervical cancer (FIGO stages: IA1 with lymphovascular space invasion, IA2, IB1 IIA1), with squamous or adenocarcinomatous histology, without suspicious images in the study of lymph node metastasis. The contraindications are suspected extrauterine involvement, presence of pathological pelvic or para-aortic lymph nodes on radiological examination, previous history of surgery or radiotherapy to nodal areas under study and contraindication for surgical treatment (related to age or associated medical conditions). Pregnancy is not a formal contraindication for SLN biopsy, even though the treatment choice for pregnant women with cervical cancer is based on FIGO stage and gestational age at diagnosis. ${ }^{21,22}$ In nursing mothers, breast-feeding should be suspended for $24 \mathrm{~h}$ after radiopharmaceutical administration. ${ }^{19}$ Kadkhodayan et al. carried out a systematic review and meta-analysis on SLN biopsy in cervical cancer and showed that, even though tracer injection in patients with previous conization might be technically challenging, they present a pooled detection rate of $91 \%$ and therefore does not represent a contraindication. Nevertheless, a history of neoadjuvant treatment (chemotherapy and/or radiotherapy), usually limited to tumors larger than $2-3 \mathrm{~cm}$ or to locally advanced disease, ${ }^{23,24}$ challenge the rate detection and accuracy of SLN biopsy, limiting the utility of this technique in such scenarios. Still, larger studies in this subject are needed. ${ }^{25}$

\section{Tracers, injection technique and detection rate}

The procedures for SLN detection and localization involve the injection of a blue dye usually combined with a 
radiopharmaceutical and/or a fluorescent agent. Subsequently, preoperative and/or intraoperative imaging can be performed. Finally, intraoperative naked eye, gamma probe or near-infrared (NIR) fluorescence imaging localization followed by surgical removal of the detected lymph nodes are performed. ${ }^{19}$

\subsection{Blue dye}

Initial studies of lymphatic mapping in patients with cervical cancer used only blue dye. Nonetheless, the detection rate ranged from $70 \%$ to $100 \% .{ }^{17}$ For this practice a water based dye such as isosulfan blue, methylene blue, or patent blue is injected into the cervix immediately after induction of anesthesia. These dyes bind weakly to plasma proteins; they are largely excreted through the biliary tract. Intraoperatively, the dye can be observed with the naked eye in both laparotomic or minimally invasive approaches. ${ }^{3}$

Several injection techniques have been described, most of them consisting in either cervical, tumoral or peritumoral. Superficial and deep injections at the 3 and 9 o'clock positions or submucosal injection into each quadrant of the cervix are the procedures most widely performed currently. ${ }^{3,17}$ All of them present common effects related to the use of blue dye: transient discoloration of the skin and urine and a decrease in pulse oximetry readings due to colorimetric interference. ${ }^{26}$ Rarely, more severe reactions, such as anaphylaxis, may occur (0.6\%). ${ }^{27,28}$ Usually blue dyes spread rapidly and arrive SLN about 5-10 min after injection. Further ahead, these substances might arrive at secondary non-SLN. Consequently, it is relevant to perform SLN biopsy at the beginning of the surgical procedure. $^{3}$

\subsection{Radioisotopes}

The interstitial injection of radioactive tracers has been widely spread in cervical cancer practice as well as in many other tumors. ${ }^{99 \mathrm{~m}}$ Technetium-sulfur colloid is the substance most commonly used in the United States, whereas ${ }^{99 m}$ Technetium-nanocolloid serum albumin is mostly used in Europe. ${ }^{12}$ The European Association of Nuclear Medicine (EANM) guidelines describe that radiopharmaceutical injection should be peritumoral or periorificial into the four quadrants of the cervix via a 20 or 22-gauge spinal needle. Usual techniques are similar to those enounced for blue dyes. In the case of previous conization, pericicatricial injection is preferred, if feasible. Instillation should be superficial (submucosal) in small tumors. In bigger tumors, injection into the necrotic part of the tumor should be carefully avoided. ${ }^{19}$ Important adverse reactions to radiocolloids have not been described thus far.

At least three protocols have been described regarding timing and doses for ${ }^{99 \mathrm{~m}} \mathrm{Tc}$ administration. Long protocols consist of cervical injection of the tracer $20-24 \mathrm{~h}$ before the surgery. Usual doses are high (2-4 mCi, 74-148 MBq) because half-life of isotope is roughly $6 \mathrm{~h}$; some institutions even repeat instillation on the day of the surgery. In short protocols radiocolloid is injected $2-4 \mathrm{~h}$ before surgery. Usual doses are between 0.2 and $1 \mathrm{mCi}$ (7.4-37 MBq). Finally, ultra-short protocols comprise injection of $0.4-0.55 \mathrm{mCi}(14.8-20 \mathrm{MBq})$ in the operating room, after induction of anesthesia (in combination with blue dye or not). In this last case preoperative images are not performed. ${ }^{29}$

Following the injection of the tracer, lymphoscintigraphy imaging is usually performed and it starts after $20-30 \mathrm{~min}$ in order to show the progression of lymphatic flow and localize SLNs. Most medical centers obtain images at 30 (early) and 60-120 min (delayed) after injection. In order to acquire planar and/or tomographic images (single photon emission computed tomography - SPECT), a gamma camera with a low-energy high-resolution collimator and single or multiple heads and a large field of view is essential. ${ }^{12}$ More recently a hybrid system with SPECT gamma camera and integrated CT scanner (SPECT/CT) that fuses tomographic lymphoscintigraphy with anatomical data has been developed. It consists of a dual-head variable-angle gamma camera armed with lowenergy high-resolution collimators and a low-intensity CT scanner, delivering a 3D image with improved spatial resolution as compared to planar imaging. This allows for the precise localization of the SLN within an anatomical background. ${ }^{19}$ When SLNs are located close to the cervix it is usually difficult to detect them since the gamma-probe picks up high activity from the injection site, which usually happens with parametrial nodes. SPECT/CT images are advantageous to distinguish these SLNs, as well as contiguous SLNs and SLNs in infrequent locations (para-aortic, presacral). ${ }^{30}$

Intraoperatively, a gamma-probe is used to acquire the radioactive signal. The probe is handled slowly and carefully in order to recognize even small SLNs in which the radiocolloid is retained. ${ }^{12}$ Lymph nodes with high gamma activity are called hot nodes. They are identified in comparison with background radioactivity (average count rates of the surrounding non-sentinel nodes and lymph node basin). The ratio of SLN counts to background counts depend not only on the relapsed time between tracer injection and surgery but also on the dose, type of tracer injected and type of gamma probe used. These ratios vary between 10:1 and 25:1. Supplementary SLN should have a counting rate of at least $20 \%$ of the counting rate in the hottest node detected. Nevertheless, residual radioactivity after exeresis must be lower than $10 \%$ of the counting rate in the hottest node. ${ }^{31}$

\subsection{Fluorescent agents}

During last years, a third option that avoids radiation exposure to patients has been raised: indocyanine green (ICG). It is a fluorescent dye classically used in ophthalmology and cardiology; it relies on NIR imaging. This dye presents high tissue penetration and low autofluorescence, not being visible to the human eye. It fluoresces in the NIR spectroscopy and this can be detected intraoperatively using a fluorescence camera. A recent meta-analysis by Ruscito et al. ${ }^{32}$ demonstrated that ICG seems to be equivalent to the combination of blue dyes and $99 \mathrm{~m}$ Tc within terms of overall and bilateral detection rates in cervical and endometrial malignancies. In a series of 227 cases collected by three US institutions, Jewel et al. communicated an overall detection rate of $95 \%$ and bilateral detection of $79 \%$ using ICG. ${ }^{33}$ No significant adverse events have been reported with ICG, except from rare anaphylactic reactions during its ophthalmic or cardiologic use. ${ }^{3}$ 
However, some authors point out that the use of ICG does not provide guidance toward unexpected areas because of the absence of preoperative images. ${ }^{34}$ In order to combine a radio-guided and a fluorescence-guided approach, a mix tracer which consists of both ${ }^{99 \mathrm{~m}} \mathrm{Tc}$-nanocolloid and ICG has been industrialized (ICG- ${ }^{99 m}$ Tc-nanocolloid). Nevertheless, Buda et al. recently published data obtained from 5 European institutions (144 patients) showing that detection rate for ICG only cases was $100 \%$ whereas for ${ }^{99 m}$ Tc combined with blue dye was $96 \%$. Moreover, bilateral mapping was accomplished in $98.5 \%$ for ICG and $76.3 \%$ for ${ }^{99 m}$ Tc with blue dye $(p<0.0001) .{ }^{35}$ They also showed that even though there was no significant difference between the two groups in terms of bilateral mapping in patients with a tumor diameter less than $2 \mathrm{~cm}$, in the case of bigger tumors ICG showed a higher bilateral mapping rate (100 vs. $64 \% ; p=0.001$ ). This evidences the possibility of avoiding the use of a radioactive agent such as ${ }^{99 \mathrm{~m}} \mathrm{Tc}$.

\subsection{Combined technique}

When blue dye and radioisotope are combined to map lymph nodes in patients with early cervical cancer, detection rates seem to be improved over those with one method alone. In their study of 211 women, Roy et al. found that the combined method of lymphatic mapping has a detection rate of $99.1 \%$, compared to $92.8 \%$ rate with blue dye alone. ${ }^{36}$ The French SENTICOL (Ganglion Sentinelle dans le Cancer du Col) prospective trial, in which a combined technique was used in 133 out of 139 cases included, at least one SLN was detected in $97.8 \%$ of patients whereas bilateral detection was accomplished in $76.5 \%$ of them. ${ }^{18}$

Many studies have pointed out that tumor size is relevant for SLN detection. Rob et al. presented an analysis of studies published on SLN biopsy in early stage cervical cancer, reporting a detection rate of $95 \%$ for tumors smaller than $2 \mathrm{~cm}$, whereas for bigger tumors, the same parameter dropped to $80 \% .{ }^{29}$ In a more recent literature review, Tax et al. revealed that blue dye and ${ }^{99 \mathrm{~m}}$ Tc combined technique shows a bilateral detection rate of $87 \%$ and $83 \%$ in patients with a tumor diameter of $<2 \mathrm{~cm}$ and $<4 \mathrm{~cm}$, respectively. ${ }^{28}$

It is important to bear in mind that the resources and equipment required for ${ }^{99 \mathrm{~m}} \mathrm{Tc}$ mapping are not accessible to all surgeons. It is costly and time consuming and demands a nuclear medicine unit with safety protocols for handling. ${ }^{12}$ Blue dye is much cheaper and generally available in most hospitals. Finally, the use of ICG is also expensive because it requires the use of specialized equipment such as fluorescence camera. ${ }^{37}$

\section{SLN mapping. Expected and unexpected drainage routes}

Based on the fact that the uterine cervix is a midline structure, several drainage routes have been described. Considering that the reasons for this distribution pattern (anatomic variations, tumor size or location, tracer used, site of injection, etc.) have not been clarified yet, the current consensus is that complete lymph node dissection must be performed on the side/s where no SLN is detected. ${ }^{38}$

Marnitz et al. claimed that exact and reproducible localization of the pelvic lymph nodes is difficult due to the absence of a uniform nomenclature for lymph nodes draining. They proposed a mapping scheme of SLNs according to their usual surgical procedure. ${ }^{39}$ Lymph nodes were labeled as para-aortic lymph nodes/area 1 (all precaval and preaortic lymph nodes up to the level of the aortic bifurcation), common iliac/area 2 (lymph nodes between the bifurcation level of the aorta and the bifurcation of the iliac vessels), external iliac/area 3 (nodes situated along the external iliac vessels, including the lymph nodes in the lumbo-sacral fossa lateral to the iliac vessels), interiliac/area 4 (lymph nodes medial to the external iliac vessels, those along the external iliac vein, lymph nodes of the obturator fossa and those covering the bifurcation of the common iliac artery), internal iliac/area 5 (nodes medial to the internal iliac vessel down to the level of the bifurcation of the uterine vessels, including the presacral lymph nodes) and finally parametric/area 6 (nodes situated at the bifurcation of the uterine vessels) as it is shown in Fig. 1. According to Marnitz's retrospective analysis of the distribution pattern for sentinel lymph nodes in 151 patients, they detected 406 sentinel lymph nodes using either a single or a combined labeling technique with ${ }^{99 \mathrm{~m}} \mathrm{Tc}$ and/or blue dye: 17 were para-aortic (4\%, area 1$)$, 20 common iliac (5\%, area 2), 20 external iliac (5\%, area 3), 288 interiliac $(71 \%$, area 4), 33 internal iliac (8\%, area 5) and 29 parametric $(7 \%$, area 6$)$. When this group used the technique of combined ${ }^{99 \mathrm{~m}} \mathrm{Tc}$ and patent blue labeling, more nodes were detected in the para-aortic area $(7 \%, p=0.006)$. The described

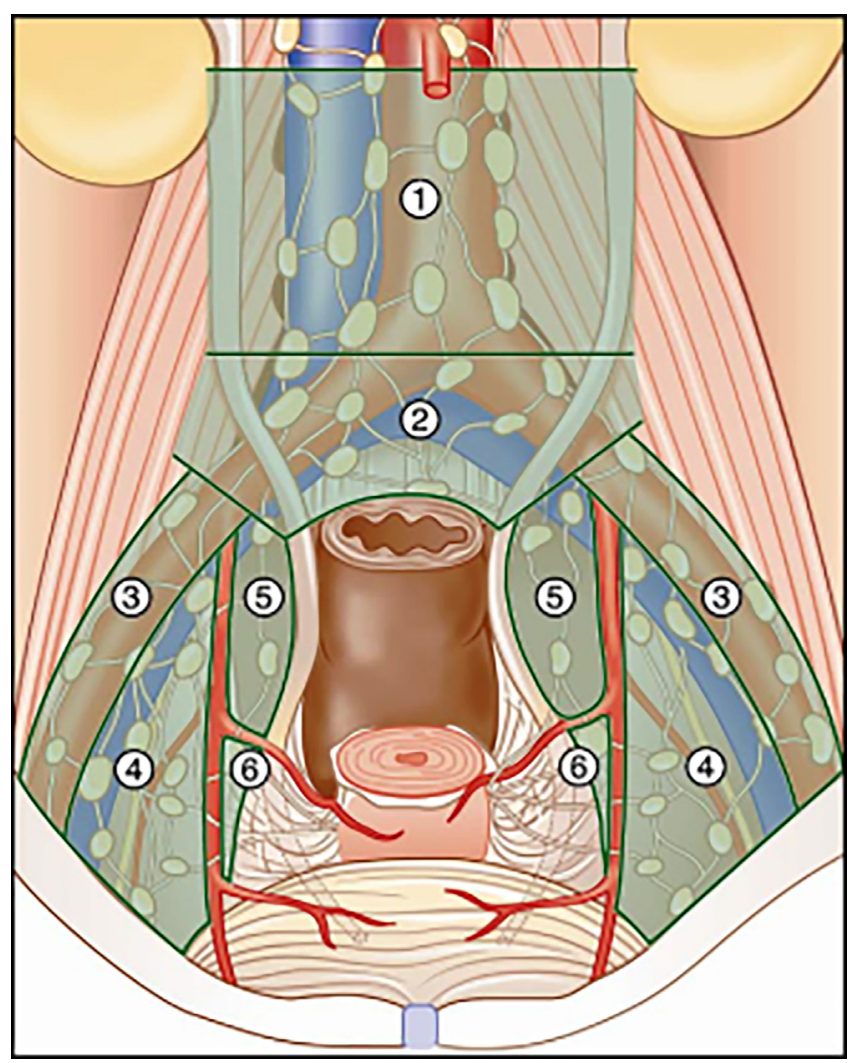

Fig. 1 - Cartographed localization of SLN by Marnitz et al. ${ }^{37}$ 
distribution implies that SLN exploration should be started at the interiliac/external iliac area (which account for more than $75 \%$ of all SLNs detected) but must also include the parametrial, presacral, iliac internal, common iliac und paraaortic areas because these are the localizations for up to $8 \%$ of SLNs. It may be observed that the prevalence of SLNs in the parametrial area can range from $0 \%$ to $15 \%$, and might constitute the first and only site of metastasis. Nevertheless, the concentration of technetium in the cervix does not permit an accurate identification of these nodes, confirming the importance of blue dye for this area. Patients with adenocarcinoma did not differ in their topographic distribution of sentinel lymph nodes from those with squamous cell cervical cancer.

Posteriorly Bats et al. validated Marnitz's mapping scheme but also pointed out that even though $80.6 \%$ of SLNs were found in areas 4 and 5, 38.2\% of patients had at least one SLN in an unexpected area and 5.1\% had SLNs only in unexpected areas. ${ }^{40}$ Areas 1, 2, 5 and 6 were defined as unexpected. This study included 139 patients with early cervical cancer. They performed SLN detection with a combined labeling technique followed by systematic pelvic lymphadenectomy as well as lymphadenectomy of areas containing one or more SLNs. They encountered SLN metastasis in $16.9 \%$ of patients with detected SLNs. Thirteen percent of these metastasis were located only in unexpected areas (para-aortic and parametrium) and they were found only by SLN biopsy.

Concern has been raised regarding the possibility of parametrial nodes (usually removed during radical hysterectomy) being the first step in cervical cancer draining routes. Both blue dye and radiocolloid are not usually highly effective to identify these nodes. Consequently, Frumovitz et al. performed a study using a triple injection of blue dye, ${ }^{99 \mathrm{~m}} \mathrm{Tc}$ and India ink. This third mapping agent stained sentinel nodes black, allowing postoperative detection and microscopic pathologic analysis in addition to usual preoperative and intraoperative identification. They included 20 patients: $25 \%$ of them showed no parametrial nodes identified on histopathological final analysis. Five patients presented metastatic disease to lymph nodes: 1 with metastasis in a parametrial node and 4 patients in pelvic nodes. Of these latter 4, one had disease that apparently skipped the ipsilateral parametrial node, whereas the other 3 had no ipsilateral parametrial lymph node identified on the side where the pelvic node presented tumor cells. This data supports that direct lymphatic drainage from the tumor to the pelvic nodes exists and it can frequently bypass the parametrial nodes, if they are present, ruling out the need for a technique that could enhance these node's detection rate. ${ }^{41}$

\section{Surgical approach}

Even though SLN biopsy can be performed via either open surgery, laparoscopy or robot-assisted surgery, it has been elicited that the two latter show a higher detection rate and sensitivity. ${ }^{25}$ Moreover, fertility-sparing techniques (conization and both simple and radical trachelectomy) are also feasible in combination with SLN biopsy. ${ }^{42,43}$

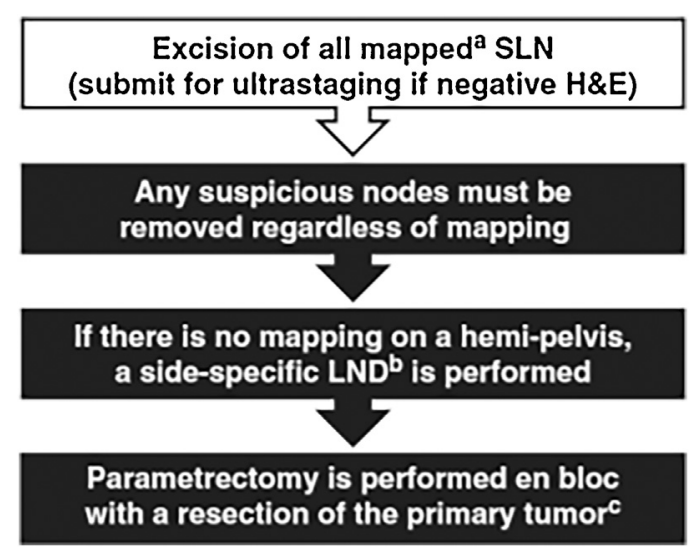

Fig. 2 - Surgical algorithm for SLN biopsy by Cormier et al. ${ }^{36}$ (a) Intracervical injection; (b) including interiliac/subaortic nodes; (c) exceptions made for select cases.

Adherence to SLN biopsy surgical algorithms is relevant and allows to achieve a low false negative-rate. Fig. 2 shows the algorithm proposed by Cormier et al. ${ }^{38}$ Direct visualization of the pelvis allows the surgical team to identify blue lymphatic channels and nodes. The following step consists in the identification of the radioactive hot nodes with either a hand-held/laparoscopic gamma-probe or fluorescent nodes via NIR. After extirpation, it is recommended to perform an ex vivo control outside the surgery field. Once SLNs are removed, it is subsequently mandatory to corroborate the presence of residual radioactivity/fluorescence emission in the pelvic and para-aortic regions. As we previously mentioned, it is important to look for parametrial SLNs based on direct visualization of blue dye or ICG due to the high radioactive emission rates near the cervix.

An essential aspect of SLN biopsy mapping is the detection of at least one SLN for each pelvic side. If no SLN is found in a hemipelvis, a side-specific lymphadenectomy must be performed. ${ }^{35}$ In order to identify suspected, enlarged nodes, a complete inspection should also be present during surgery. Bulky, massively infiltrated lymph nodes can impair their detection, regardless the tracer used. These nodes cannot be identified as sentinel nodes (probably due to metastatic blockage) and should not be eluded: they must be removed and sent for intraoperative pathological analysis regardless of the SLN mapping. ${ }^{16}$

If intraoperative pathological study shows a positive node, proper treatment is based on chemo-radiation. Therefore, hysterectomy might be omitted in order to avoid unnecessary morbidity without increasing overall survival, ${ }^{19}$ although prospective randomized studies on the need of performing or abandoning radical hysterectomy are still required. ${ }^{44}$ The usefulness of undertaking a systematic para-aortic (with or without pelvic) lymphadenectomy in order to tailor the radiation field, following findings on locally advanced cervical cancer, ${ }^{45}$ remains controversial, ${ }^{19}$ although some authors have elicited that this would not represent an added benefit on overall survival. ${ }^{46}$ 


\section{Pathological analysis/ultrastaging}

Characteristically frozen section has been used for the evaluation of SLNs. Nevertheless, several studies have found that the precision of intraoperative frozen section evaluation to detect micrometastasis, and small macrometastasis is limited. ${ }^{3}$ Slama et al. described that overall sensitivity of frozen section analysis was $56 \%$ for all types of metastasis, mainly due to its inability to detect low volume disease. ${ }^{47}$ In this series of 225 patients, frozen section was only able to detect 2 out of 17 cases of micrometastasis and none of 8 cases with isolated tumor cells (ITC).

Pathologic ultrastaging includes multiple serial sectioning and immunohistochemical assessment of SLNs. It permits the detection of low-volume metastasis of less than $2 \mathrm{~mm}$, including both micrometastasis $(0.2-2 \mathrm{~mm}$ ) and ITC (less than $0.2 \mathrm{~mm}$ ). In a large retrospective cohort study with 645 patients, macrometastasis (tumor deposits larger than $2 \mathrm{~mm}$ ), micrometastasis and ITC were detected by ultrastaging and staging of all other pelvic lymph nodes in $21 \%$, $7 \%$, and $4 \%$ of cases, respectively. ${ }^{48}$ The sensitivity of SLN ultrastaging reached $91 \%$ in the entire group and $97 \%$ in patients with bilateral SLN detection, with a false negative rate of $1.3 \%$. This allows to identify nodal involvements that would have never been detected with standard techniques.

Furthermore, according to the analysis of the same cohort made by Cibula et al., the overall survival of patients with micrometastasis (7.1\% of the sample) was similar to patients with macrometastasis, and this was statistically significant compared to patients with negative SLNs $(p<0.001) .{ }^{49}$ The presence of micrometastasis was an independent prognostic factor for survival. No prognostic significance was found for ITC.

The one factor that was significantly more frequent in a subgroup with false negative results was the presence of lymphovascular space invasion (not age, histological type, number of removed SLN nor tumor stage), but this constituted a very small group to make a strong conclusion. Regardless of the broad evidence regarding ultrastaging on SLN biopsy in cervical cancer, it is still scarcely used in current clinical management. ${ }^{49}$

\section{Clinical relevance of SLN biopsy as an alternative to lymphadenectomy}

In order to weigh SLN biopsy trustworthiness, sensitivity and false negative rate are crucial. They assess the possibility of metastatic involvement of the rest of pelvic lymph nodes when SLNs are negative. There is good evidence of the high sensitivity of SLN biopsy concept in cervical cancer. To the best of our knowledge, most of the evidence arises from retrospective trials and one prospective trial.

Lécuru et al. published the formerly referred SENTICOL prospective multicenter study with 139 patients that presented IA1 to IB1 cervical cancer. They applied SLN ultrastaging, obtaining a sensitivity of $92 \%$ (95\% CI, 74.0-99\%) for the whole group and $100 \%$ in patients with bilateral SLN detection. ${ }^{18}$ Negative predictive value (NPV) was $98.2 \%$ (95\% CI,
93.8-99.8\%) for the whole sample. As previously mentioned, Cibula et al. published the largest retrospective multicenter study to the date and registered 645 stage IA1 to IIB patients, also performing ultrastaging. They showed an overall sensitivity of $91 \%$ and $97 \%$ for patients with bilateral detection. ${ }^{48}$ Sensitivity was not compromised in larger tumors or in higher stages in patients with bilateral SLN detection.

Tax et al. performed a literature review that included 47 studies reporting results on SLN detection, in patients with early stage cervical cancer, totaling 4130 cases. When considering only ultrastaging studies, they obtained a sensitivity of $94 \%$ and a NPV range of $91-100 \%$ (compared to a sensitivity of $68 \%$ and NPV range of $59-100 \%$ from frozen section and/or H\&E analysis). Ultrastaging showed 19 false negative cases out of 1275 patients, with a $1.5 \%$ consequent risk of undertreatment. Nevertheless, the author explored the characteristics of these 19 patients, concluding that adding more specific criteria for the selection of cases, 18 cases would have been prevented, decreasing the chance of undertreatment to $0.08 \%(1 / 1257)$. Such criteria were: (1) bilateral detection, (2) early FIGO stages IA2, IB1, IIA, (3) no suspicious lymph nodes during either pre-operative imaging or surgery and (4) primary tumor size smaller than $40 \mathrm{~mm}$. Overall, when the latter criteria were met, estimated sensitivity was $99.6 \%$ (95\% CI: 98-100\%) and NPV $99.9 \%$. In melanoma, breast and vulvar cancer, the remaining risk of $3 \%, 0-10 \%$ and $2 \%$ of metastasis in non-SLN have been considered adequate to abandon lymphadenectomy. ${ }^{28}$

SLN biopsy not only reduces surgical time, blood loss, rate of infection, lymphocele and lymphedema but it can also improve detection of lymph node metastasis compared to lymphadenectomy. ${ }^{50}$ Aberrant lymphatic drainage pathways, positive evidence of lymph node removal, and ultrastaging enhance the power of this technique. ${ }^{11}$ Currently, a prospective observational trial on sentinel lymph node biopsy in patients with early stage cervical cancer, the SENTIX (SENTinel lymph node in cervIX cancer) trial (CEEGOG CX-01; ENGOT-CX 2; NCT02494063) is in recruitment phase. It is the first prospective trial willing to evaluate whether SLN biopsy is non-inferior to treatment with systematic pelvic lymphadenectomy.

\section{Conclusion}

Lymph node status remains a main factor for the prognosis and management of cervical cancer. The use of SLN biopsy in clinical practice is justified by the lower number of unnecessary lymph node dissections and its consequences in terms of surgical time, complications and posterior morbidity. Moreover, an additional benefit from SLN includes a better information about nodal status (unusual drainage sites) and a high rate of low-volume metastasis detection (ultrastaging). Nowadays, SLN biopsy represents a cornerstone in the management of early stage cervical cancer patients by multidisciplinary trained teams in validated centers.

\section{Conflict of interest}

None declared. 


\section{Financial disclosure}

None declared.

\section{Acknowledgements}

The authors thank Prof. Àngels Rovirosa for her trust and support.

\section{R E F E R E N C E S}

1. Ferlay J, Soerjomataram I, Ervik M, et al. GLOBOCAN 2012 v1.0, cancer incidence and mortality worldwide: IARC CancerBase No. 11. Lyon: Fr Int Agency Res Cancer; 2013. Available from http://www.globocan.iarc.fr [last accessed 01/08/17].

2. Pecorelli S. Revised FIGO staging for carcinoma of the vulva, cervix, and endometrium. Int J Gynecol Obstet 2009;105:103-4.

3. Holman LL, Levenback CF, Frumovitz M. Sentinel lymph node evaluation in women with cervical cancer. J Minim Invasive Gynecol 2014;21(July (4)):540-5.

4. Kim SM, Choi HSBJ. Overall 5-year survival rate and prognostic factors in patients with stage IB and IIA cervical cancer treated by radical hysterectomy and pelvic lymph node dissection. Int J Gynecol Cancer 2000;10(4):305-12.

5. National Comprehensive Cancer Network (NCCN). Clinical practice guidelines in oncology. Cervical cancer version I; 2017. October 10 2016. Available from: https://www.nccn. org/professionals/physician_gls/pdf/cervical.pdf [last accessed 10/01/17].

6. Sartori E, Tisi G, Chiudinelli F, La Face B, Franzini R, Pecorelli S. Early stage cervical cancer: adjuvant treatment in negative lymph node cases. Gynecol Oncol 2007;107(1 Suppl. 1):170-4.

7. Bipat S, Glas AS, van der Velden J, Zwinderman AH, Bossuyt PMM, Stoker J. Computed tomography and magnetic resonance imaging in staging of uterine cervical carcinoma: a systematic review. Gynecol Oncol 2003;91(1):59-66.

8. Khiewvan B, Torigian DA, Emamzadehfard S, et al. Update of the role of PET/CT and PET/MRI in the management of patients with cervical cancer. Hell J Nucl Med 2016;19(3):254-68.

9. Sakuragi N. Up-to-date management of lymph node metastasis and the role of tailored lymphadenectomy in cervical cancer. Int J Clin Oncol 2007;12:165-75.

10. Achouri A, Huchon C, Bats AS, Bensaid C, Nos C, Lécuru F. Complications of lymphadenectomy for gynecologic cancer. Eur J Surg Oncol 2013;39:81-6.

11. Lennox GK, Covens A. Can sentinel lymph node biopsy replace pelvic lymphadenectomy for early cervical cancer? Gynecol Oncol 2017;144(January (1)):16-20.

12. Palla V-V, Karaolanis G, Moris D, Antsaklis A. Sentinel lymph node biopsy in uterine cervical cancer patients: ready for clinical use? A review of the literature. ISRN Surg 2014;2014(January):841618.

13. Wang X-J, Fang F, Li Y-F. Sentinel-lymph-node procedures in early stage cervical cancer: a systematic review and meta-analysis. Med Oncol 2015;32(January (1)):385.

14. Bats A-S, Frati A, Froissart M, et al. Feasibility and performance of lymphoscintigraphy in sentinel lymph node biopsy for early cervical cancer: results of the prospective multicenter SENTICOL study. Ann Nucl Med 2015;29(January (1)):63-70.

15. Cabanas RM. An approach for the treatment of penile carcinoma. Cancer 1977;39(February (2)):456-66.
16. O’Boyle JD, Coleman RL, Bernstein SG, Lifshitz S, Muller CY, Miller DS. Intraoperative lymphatic mapping in cervix cancer patients undergoing radical hysterectomy: a pilot study. Gynecol Oncol 2000;79(November (2)):238-43.

17. Dargent D, Enria R. Laparoscopic assessment of the sentinel lymph nodes in early cervical cancer. Technique preliminary results and future developments. Crit Rev Oncol Hematol 2003;48(December (3)):305-10.

18. Lécuru F, Mathevet P, Querleu D, et al. Bilateral negative sentinel nodes accurately predict absence of lymph node metastasis in early cervical cancer: results of the SENTICOL study. J Clin Oncol 2011;29(May (13)):1686-91.

19. Giammarile F, Bozkurt MF, Cibula D, et al. The EANM clinical and technical guidelines for lymphoscintigraphy and sentinel node localization in gynaecological cancers. Eur J Nucl Med Mol Imaging 2014;41(July (7)):1463-77.

20. Khoury-Collado F, Glaser GE, Zivanovic O, et al. Improving sentinel lymph node detection rates in endometrial cancer: how many cases are needed? Gynecol Oncol 2009;115(December (3)):453-5.

21. Silva LB, Silva-Filho AL, Traiman P, et al. Sentinel node mapping in a pregnant woman with cervical cancer: a case report. Int J Gynecol Cancer 2006;16(May (3)):1454-7.

22. Papadia A, Mohr S, Imboden S, Lanz S, Bolla D, Mueller MD. Laparoscopic indocyanine green sentinel lymph node mapping in pregnant cervical cancer patients. J Minim Invasive Gynecol 2016;23(February (2)):270-3.

23. Plante M. Evolution in fertility-preserving options for early-stage cervical cancer radical trachelectomy, simple trachelectomy, neodjuvant chemotherapy. Int J Gynecol Cancer 2013;23(6):982-9.

24. Slama J, Dundr P, Dusek L, et al. Sentinel lymph node status in patients with locally advanced cervical cancers and impact of neoadjuvant chemotherapy. Gynecol Oncol 2012;125(2): 303-6.

25. Kadkhodayan S, Hasanzadeh M, Treglia G, et al. Sentinel node biopsy for lymph nodal staging of uterine cervix cancer: a systematic review and meta-analysis of the pertinent literature. Eur J Surg Oncol 2015;41(January (1)):1-20.

26. Vieira SC, Sousa RB, Tavares MBAC, et al. Changes in pulse oximetry after patent blue dye injection into the uterine cervix. Ann Surg Oncol 2008;15(October (10)):2862-6.

27. Bricou A, Barranger E, Uzan S, Darai E. Anaphylactic shock during the sentinel lymph node procedure for cervical cancer. Gynecol Oncol 2009;114(August (2)):375-6.

28. Tax C, Rovers MM, de Graaf C, Zusterzeel PLM, Bekkers RLM. The sentinel node procedure in early stage cervical cancer, taking the next step; a diagnostic review. Gynecol Oncol 2015;139(December (3)):559-67.

29. Rob L, Robova H, Halaska Michael J, Hruda M, Skapa P. Current status of sentinel lymph node mapping in the management of cervical cancer. Expert Rev Anticancer Ther 2013;13(July (7)):861-70.

30. Collarino A, Vidal-Sicart S, Perotti G, Valdés Olmos RA. The sentinel node approach in gynaecological malignancies. Clin Transl Imaging 2016;4(October (5)):411-20.

31. Darlin L, Persson J, Bossmar T, et al. The sentinel node concept in early cervical cancer performs well in tumors smaller than $2 \mathrm{~cm}$. Gynecol Oncol 2010;117(May (2)):266-9.

32. Ruscito I, Gasparri ML, Braicu EI, et al. Sentinel node mapping in cervical and endometrial cancer: indocyanine green versus other conventional dyes - a meta-analysis. Ann Surg Oncol 2016;23(October (11)):3749-56.

33. Jewell EL, Huang JJ, Abu-Rustum NR, et al. Detection of sentinel lymph nodes in minimally invasive surgery using indocyanine green and near-infrared fluorescence imaging for uterine and cervical malignancies. Gynecol Oncol 2014;133(May (2)):274-7. 
34. Paredes P, Vidal-Sicart S, Campos F, et al. Role of ICG-99m Tc-nanocolloid for sentinel lymph node detection in cervical cancer: a pilot study. Eur J Nucl Med Mol Imaging 2017;44(October (11)):1853-61.

35. Buda A, Papadia A, Zapardiel I, et al. From conventional radiotracer Tc-99m with blue dye to indocyanine green fluorescence: a comparison of methods towards optimization of sentinel lymph node mapping in early stage cervical cancer for a laparoscopic approach. Ann Surg Oncol 2016;23(September (9)):2959-65.

36. Roy M, Bouchard-Fortier G, Popa I, et al. Value of sentinel node mapping in cancer of the cervix. Gynecol Oncol 2011;122(August (2)):269-74.

37. Cormier B, Rozenholc AT, Gotlieb W, Plante M, Giede C. Sentinel lymph node procedure in endometrial cancer: a systematic review and proposal for standardization of future research. Gynecol Oncol 2015;138(August (2)):478-85.

38. Cormier B, Diaz JP, Shih K, et al. Establishing a sentinel lymph node mapping algorithm for the treatment of early cervical cancer. Gynecol Oncol 2011;122(August (2)):275-80.

39. Marnitz S, Köhler C, Bongardt S, Braig U, Hertel H, Schneider A. Topographic distribution of sentinel lymph nodes in patients with cervical cancer. Gynecol Oncol 2006;103(October (1)):35-44.

40. Bats A-S, Mathevet P, Buenerd A, et al. The sentinel node technique detects unexpected drainage pathways and allows nodal ultrastaging in early cervical cancer: insights from the multicenter prospective SENTICOL study. Ann Surg Oncol 2013;20(February (2)):413-22.

41. Frumovitz M, Euscher ED, Deavers MT, et al. "Triple injection" lymphatic mapping technique to determine if parametrial nodes are the true sentinel lymph nodes in women with cervical cancer. Gynecol Oncol 2012;127(December (3)):467-71.

42. Kim CH, Soslow RA, Park KJ, et al. Pathologic ultrastaging improves micrometastasis detection in sentinel lymph nodes during endometrial cancer staging. Int J Gynecol Cancer 2013.

43. Du X-L, Sheng X-G, Jiang T, et al. Sentinel lymph node biopsy as guidance for radical trachelectomy in young patients with early stage cervical cancer. BMC Cancer 2011;11(December (1)):157.

44. Derks M, Groenman FA, van Lonkhuijzen LRCW, et al. Completing or abandoning radical hysterectomy in early-stage lymph node Y positive cervical cancer impact on disease-free survival and treatment-related toxicity. Int J Gynecol Cancer 2017;27(5):1015-20.

45. Del Pino M, Fusté P, Pahisa J, et al. Laparoscopic lymphadenectomy in advanced cervical cancer. Prognostic and therapeutic value. Int J Gynecol Cancer 2013;23:1675-83.

46. Barquet-Muñoz SA, Rendón-Pereira GJ, Acuña-González D, et al. Role of pelvic and para-aortic lymphadenectomy in abandoned radical hysterectomy in cervical cancer. World J Surg Oncol 2017;15(23):1-9.

47. Slama J, Dundr P, Dusek L, Cibula D. High false negative rate of frozen section examination of sentinel lymph nodes in patients with cervical cancer. Gynecol Oncol 2013;129(May (2)):384-8.

48. Cibula D, Abu-Rustum NR, Dusek L, et al. Bilateral ultrastaging of sentinel lymph node in cervical cancer: lowering the false-negative rate and improving the detection of micrometastasis. Gynecol Oncol 2012;127(December (3)):462-6.

49. Cibula D, Abu-Rustum NR, Dusek L, et al. Prognostic significance of low volume sentinel lymph node disease in early-stage cervical cancer. Gynecol Oncol 2012;124(March (3)):496-501.

50. Gortzak-Uzan L, Jimenez W, Nofech-Mozes S, et al. Sentinel lymph node biopsy vs. pelvic lymphadenectomy in early stage cervical cancer: is it time to change the gold standard? Gynecol Oncol 2010;116(January (1)):28-32. 